\title{
Reducing ASE Effect in Coherent Detection by Employing Double-Pass Fiber Preamplifier and Time-Domain Filter
}

\author{
Youming Chen, Brian L. Mark, Senior Member, IEEE, Ralph Burnham, and Horacio Verdun, Member, IEEE
}

\begin{abstract}
We report on a novel fiber based coherent detection system employing a double-pass fiber preamplifier, a spectral bandpass filter, and a time-domain filter. The time-domain filter, a synchronous time gate, reduces the in-band amplified spontaneous emission (ASE) beat noise, which cannot be achieved by the spectral bandpass filter alone. The double-pass fiber amplifier further reduces the out-band $A S E$ by about $20 \mathrm{~dB}$ with a fiber Bragg grating (FBG) at the end of the fiber amplifier. In preliminary experiments with a $100 \mathrm{GHz}$ bandpass filter, no degradation is observed from the optically preamplified coherent detection compared to pure coherent detection. With a $10 \mathrm{~ns}$ pulse width, $500 \mathrm{kHz}$ repetition rate, and $10 \mathrm{pW}$ average input power, about $2 \mathrm{~dB}$ and $1 \mathrm{~dB}$ signal-to-noise ratio (SNR) improvements are achieved when $5 \%$ and $50 \%$ time gating duty cycles are used, respectively.
\end{abstract}

Index Terms-Coherent detection, detection, double-pass fiber preamplifier, sensitivity, time-domain filter (TDF).

\section{INTRODUCTION}

$\mathbf{T}$ HE sensitivity of signal detection is of major interest for optical high speed communication systems and LIght Detection And Ranging (lidar) systems. Sensitive receivers in fiber-optical networks can reduce transmitter power or amplifier amplification requirements and extend link spans. High receiver sensitivity allows links to be established over long distances in deep space satellite communication systems and large atmospheric attenuation to be overcome in terrestrial free space communications. For lidar systems, the sensitivity of signal detection determines how far and how accurately the lidar can detect remote objects.

Optical receivers employ either coherent or direct detection. In addition to amplitude, coherent detection extracts frequency and phase information from received signals, whereas direct detection extracts the received pulse amplitude only. Theory and previous experiments suggest that coherent detection should yield higher receiver sensitivity than direct detection [1], [2]. Another approach to improve detection sensitivity is to employ a fiber preamplifier. This technique has been successfully demonstrated in direct detection systems [3]-[6], but not in coherent detection systems. Due to the existence of amplified spontaneous emission (ASE) inside the amplifier, the sensitivity of

Manuscript received July 14, 2008; revised January 26, 2009. Current version published September 23, 2009.

Y. Chen, R. Burnham, and H. Verdun are with Fibertek, Inc., Vienna, VA 22181 USA.

B. L. Mark is with the Department of Electrical and Computer Engineering, George Mason University, Fairfax, VA 22030 USA (e-mail: bmark@gmu.edu).

Digital Object Identifier 10.1109/JQE.2009.2024773 coherent detection is degraded when the system operates close to the shot noise limited condition [7]. For this reason, fiber based optically preamplified coherent detection is not used in applications as commonly as direct detection. However, if a coherent system is difficult to operate under a shot noise limited condition, the use of an optical amplifier with low noise figure in terms of a preamplifier will effectively improve the performance of the receiver. This situation has been investigated in [8] for a continuous phase frequency shift keying (CPFSK) system. The sensitivity assessment is also discussed in [9] for the application of an erbium doped fiber amplifier (EDFA) in a coherent receiver using pulse position modulation (PPM). The paper demonstrated significant improvement by using an optical preamplifier when the system operates under a thermal noise dominant condition.

In this paper, ${ }^{1}$ we report on a new architecture for a coherent detection system, which employs a double-pass fiber preamplifier and a time-domain filter (TDF). The TDF was used successfully in a fiber preamplified direct detection system in [6]. The main motivation for this research arises from the need for highly sensitive detection in lidar applications. The TDF is especially effective in pulsed coherent lidar systems, in which the signal has a relatively low duty cycle. To the best of our knowledge, the present paper is the first to evaluate the performance of a double-pass preamplified coherent detection system. Previous papers (cf. [11]-[15]) study the amplification properties of the double-pass EDFA, but they do not provide a system performance evaluation. The experimental investigation presented in this paper has been made possible by the recent availability of the polarization-maintaining (PM) fiber Bragg grating (FBG) and the PM circulator.

We first describe the system architecture design and provide a theoretical noise analysis. Then, we present simulation results for the performance of the double-pass fiber preamplifier. Next, we present our experimental results, including results for direct detection, and coherent detection with and without the timedomain filter. Discussions and conclusions are given in the final section.

\section{OPtICAL PREAMPLIFIEd COHERENT DeteCtion SYSTEM}

\section{A. Principle of the Coherent Detection System}

A schematic diagram of the optical preamplified coherent detection system extended by using time domain and frequency sults.

\footnotetext{
${ }^{1}$ This paper is an extended version of [10], including new experimental re-
} 


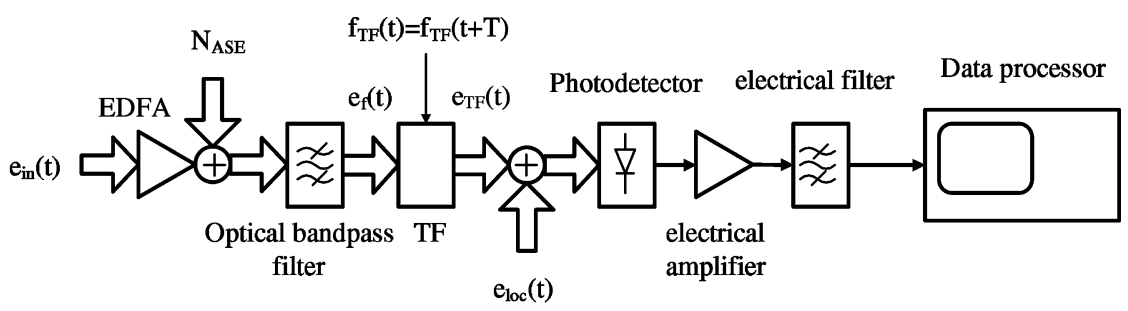

Fig. 1. Schematic diagram of optically preamplified coherent detection employing a time-domain filter.

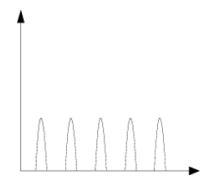

a.

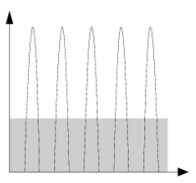

b.

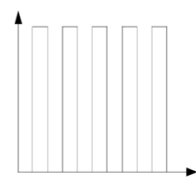

c.

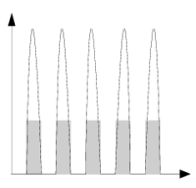

d.
Fig. 2. Pulse development in the time domain. (a) original input pulses; (b) output of the amplifier, with added ASE; (c) the time-domain filter; (d) output of the time-domain filter.

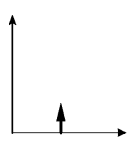

a.

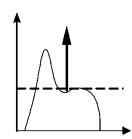

b.

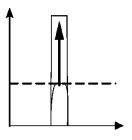

c.

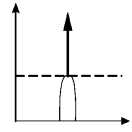

d.

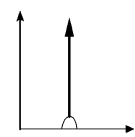

e.
Fig. 3. The spectrum properties in the frequency domain obtained by applying a narrowband filter. (a) original input signal; (b) output of amplifier, with added ASE; (c) narrow passband filter; (d) output of passband filter; (e) output of the time-domain filter.

domain filters is shown in Fig. 1. The optical signals processed at different stages in the time and frequency domains are shown in Figs. 2 and 3, respectively.

The input optical pulse trains are amplified when the pulses pass through the active optical preamplifier. Meanwhile, these amplified optical pulses are also corrupted by ASE. The ASE added to the pulses will degrade the optical signal-to-noise ratio (SNR) of the detection system [see Figs. 2(b) and 3(b)]. A spectral bandpass filter is used to filter out the unnecessary spectrum components outside of the band of the central wavelength [see Fig. 3(c) and (d)]. Then, the amplified and filtered pulses are fed through the time-domain filter as shown in Fig. 2(c) and (d). The length of the time window can be varied according to the needs of the application. The time-domain filter combined with the narrowband spectrum filter can significantly reduce the negative effects caused by ASE on coherent detection performance [see Fig. 3(e)].

\section{B. Noise Analysis}

As shown in Fig. 1, when an arbitrary, complex, optical field $e_{\text {in }}(t)$ is preamplified by a low noise amplifier with small signal gain of $G$, the preamplified signal will be additionally corrupted by ASE. Since the ASE is treated as an additive Gaussian process $n_{\mathrm{ASE}}(t)$, after amplification, the optical field becomes

$$
e_{G}(t)=\sqrt{G} e_{\mathrm{in}}(t)+n_{\mathrm{ASE}}(t) .
$$

The preamplified optical field is spectrally filtered by an optical bandpass filter with impulse response function $h_{o}(t)$. The filtered optical field $e_{f}(t)$ becomes

$$
e_{f}(t)=\left[\sqrt{G} e_{\mathrm{in}}(t)+n_{\mathrm{ASE}}(t)\right] * h_{o}(t) .
$$

Then, the optical field is temporally filtered by the TDF with a periodic function $f_{T F}(t)=f_{T F}(t+T)$, where $T$ is the period. To simplify the analysis, the TDF function can be written as

$$
f_{T F}(t)= \begin{cases}1, & 0 \leq t \leq D \\ 0, & D<t \leq T\end{cases}
$$

where $D$ is the gate width. The TDF filtered field becomes a cyclostationary process $e_{T F}(t)=e_{f}(t) \cdot f_{T F}(t)$. Upon reaching this point, the optical field mixes with the local oscillator field $e_{\text {loc }}(t)$ at the photodetector. The optical field can be further written as

$$
e_{\text {mix }}(t)=\left\{\left[\sqrt{G} e_{\mathrm{in}}(t)+n_{\mathrm{ASE}}\right] * h_{o}(t)\right\} \cdot f_{T F}(t)+e_{\mathrm{loc}}(t) .
$$

According to the square law, the detected signal current after the electrical filter is given by [16]

$$
\langle i(t)\rangle=\eta\left\langle\left|e_{\operatorname{mix}}(t)\right|^{2}\right\rangle * h_{e}(t)
$$

where $\eta$ denotes the overall optoelectronic conversion factor of the detection system; $h_{e}(t)$ is the electrical transfer function of the photodetector, electrical amplifier, and electrical bandpass filter. Applying (4) into (5), we have

$$
\begin{aligned}
\langle i(t)\rangle= & \eta\left[G p_{\text {ino }}(t) * h_{e}(t)\right]+\eta N_{\mathrm{ASEo}}\left[f_{T F}^{2}(t) * h_{e}(t)\right] \\
& +\eta\left[p_{\mathrm{loc}}(t) * h_{e}(t)\right]+2 \eta\left[\sqrt{G p_{\text {ino }}(t) p_{\mathrm{loc}}(t)} * h_{e}(t)\right] \\
& +2 \eta\left[\sqrt{G p_{\text {ino }}(t) N_{\mathrm{ASEo}}} * h_{e}(t)\right] \\
& +2 \eta\left[\sqrt{G p_{\mathrm{loc}}(t) N_{\mathrm{ASEo}}} * h_{e}(t)\right]
\end{aligned}
$$

where $p_{\text {ino }}(t)=\left\langle\left|e_{\text {in }}(t) * h_{o}(t)\right|^{2}\right\rangle$ represents the input signal power after the optical filter, $N_{\mathrm{ASEo}}=\left\langle\left|n_{\mathrm{ASE}}(t) * h_{o}(t)\right|^{2}\right\rangle$ is the average ASE power after filtering with optical bandwidth $B_{o}$, and $p_{\text {loc }}(t)=\left\langle\left|e_{\text {loc }}(t)\right|^{2}\right\rangle$ is the local oscillation power. The variance of the photocurrent is given by

$$
\begin{aligned}
\sigma^{2}(t)= & \eta q\left\langle\left|e_{\text {mix }}(t)\right|\right\rangle^{2} * h_{e}{ }^{2}(t) \\
& +\eta^{2} \iint_{-\infty}^{\infty}\left\langle\left|e_{\text {mix }}(\tau)\right|^{2}\left|e_{\text {mix }}(\tilde{\tau})\right|^{2}\right\rangle \\
& -\left\langle\left|e_{\text {mix }}(\tau)\right|^{2}\right\rangle\left\langle\left|e_{\text {mix }}(\tilde{\tau})\right|^{2}\right\rangle h_{e}(t-\tau) h_{e}(t-\tilde{\tau}) d t d \tilde{\tau}
\end{aligned}
$$


where $q$ denotes the elementary charge. The first term on the right-hand side of (7) is the variance of the shot noise and the second term is the beat noise [17]. After applying the moment theorem of Gaussian processes and doing some algebra, the variance can be written as

$$
\sigma_{\mathrm{det}}^{2}=\sigma_{\mathrm{shot}}^{2}+\sigma_{\mathrm{LOC}-\mathrm{ASE}}^{2}+\sigma_{s-\mathrm{ASE}}^{2}+\sigma_{\mathrm{ASE}-\mathrm{ASE}}^{2}+\sigma_{\mathrm{th}}^{2} .
$$

The variance of the shot noise caused by the signal, ASE, and local oscillation is given by

$$
\sigma_{\text {shot }}^{2}(t)=\eta q\left[G p_{\text {ino }}(t)+N_{\mathrm{ASE}} f_{T F}^{2}(t)+p_{\mathrm{loc}}(t)\right] * h_{e}^{2}(t) .
$$

The variance of the signal-ASE beating noise is given by

$$
\begin{gathered}
\sigma_{s-\mathrm{ASE}}^{2}(t)=2 \eta^{2} G \mathcal{R}\left\{\iint_{-\infty}^{\infty}\left|f_{T F}(\tau)\right|\left|f_{T F}(\tilde{\tau})\right| e_{\mathrm{ino}}(\tau) e_{\mathrm{ino}}^{*}\right. \\
\left.\times(\tilde{\tau})\left\langle n_{\mathrm{ASEo}}(t) n_{\mathrm{ASEo}}^{*}(\tilde{t})\right\rangle h_{e}(t-\tau) h_{e}(t-\tilde{\tau}) d t d \tilde{\tau}\right\}
\end{gathered}
$$

where $\mathcal{R}\{\cdot\}$ denotes the real part. The variance of the ASE-ASE beating noise is given by

$$
\begin{aligned}
\sigma_{\mathrm{ASE}-\mathrm{ASE}}^{2}(t)= & \eta^{2} \iint_{-\infty}^{\infty}\left|f_{T F}(\tau)\right|^{2}\left|f_{T F}(\tilde{\tau})\right|^{2} \mid\left\langle n_{\mathrm{ASE}}(t)\right. \\
& \left.\times n_{\mathrm{ASE}}^{*}(\tilde{t})\right\rangle\left.\right|^{2} h_{e}(t-\tau) h_{e}(t-\tilde{\tau}) d t d \tilde{\tau} .
\end{aligned}
$$

The variance of the local oscillation-ASE beating noise is given by

$$
\begin{aligned}
& \sigma_{\mathrm{loc}-\mathrm{ASE}}^{2}(t)=2 \eta^{2} \mathcal{R}\left\{\iint_{-\infty}^{\infty}\left|f_{T F}(\tau)\right|\left|f_{T F}(\tilde{\tau})\right| e_{\mathrm{loc}}(\tau)\right. \\
& \left.\times e_{\mathrm{loc}}^{*}(\tilde{\tau})\left\langle n_{\mathrm{ASEo}}(t) n_{\mathrm{ASEo}}^{*}(\tilde{t})\right\rangle h_{e}(t-\tau) h_{e}(t-\tilde{\tau}) d t d \tilde{\tau}\right\} .
\end{aligned}
$$

If the bandwidth $B_{o}$ of the optical filter is much wider than that of the electrical filter, $B_{e}$, and the cyclostationary process is taken into account, (9)-(12) can be simplified as follows. The variance of the shot noise becomes

$$
\sigma_{\text {shot }}^{2}(t)=\eta q\left[G p_{\text {ino }}(t)+N_{\text {ASEo }}\left(\frac{D}{T}\right)^{2}+p_{\text {loc }}(t)\right] * h_{e}^{2}(t) .
$$

The variance of the signal-ASE beating noise becomes

$$
\sigma_{s-\mathrm{ASE}}^{2}(t)=2 \eta^{2} G N_{\mathrm{ASEo}}\left(\frac{D}{T}\right)^{2}\left[p_{\text {ino }}(t) * h_{e}^{2}(t)\right] .
$$

The variance of the ASE-ASE beating noise becomes

$$
\sigma_{\mathrm{ASE}-\mathrm{ASE}}^{2}(t)=\eta^{2} N_{\mathrm{ASEo}}^{2}\left(\frac{D}{T}\right)^{4} .
$$

The variance of the local oscillation-ASE beating noise becomes

$$
\sigma_{\text {loc-ASE }}^{2}(t)=2 \eta^{2} N_{\text {ASEo }}\left(\frac{D}{T}\right)^{2}\left[p_{\text {loc }}(t) * h_{e}^{2}(t)\right] .
$$

The receiver electrical signal-to-noise ratio can be represented as the ratio of the photocurrent to the photocurrent variance:

$$
\begin{aligned}
\mathrm{SNR} & =\frac{I_{s}^{2}}{\sigma_{\mathrm{det}}^{2}} \\
& =\frac{2 \eta G p_{\mathrm{loc}} p_{\mathrm{ino}}}{\sigma_{\mathrm{shot}}^{2}+\sigma_{s-\mathrm{ASE}}^{2}+\sigma_{\mathrm{ASE}-\mathrm{ASE}}^{2}+\sigma_{\mathrm{loc}-\mathrm{ASE}}^{2}+\sigma_{\mathrm{th}}^{2}} .
\end{aligned}
$$

This is the general equation that can be used for detection evaluation and noise analysis. If $D$ equates to $T$, the system reduces to the case of optically preamplified coherent detection without the time-domain filter. If additionally $N_{\mathrm{ASEo}}$ equates to zero, $D$ equates to $T$, and $G$ equates to one, the system becomes equivalent to regular coherent detection without an optical amplifier. From (13) to (16), we can see that the ASE power affects all the variances of detection signals of interest; therefore, a narrow optical bandpass filter can effectively improve the detection performance by reducing the ASE power. From the above equations, it is also clear that the smaller the duty cycle $(D / T)$, the smaller the variance of the detection system.

\section{Theoretical Analysis of Double-Pass FIBER OPTICAL PREAMPLIFIER}

From the above noise analysis, we can see that ASE is an important issue in optical amplifiers. The ASE effect occurs concurrently with signal amplification and degrades the SNR. To reduce the ASE effect, achieve high gain and low noise figure, double-pass amplifier configurations have been experimentally investigated in [11]-[13]. In the double-pass amplifier, the signal propagates bidirectionally in the gain medium fiber, in which the amplification occurs twice. Compared to a single-pass amplifier, the double-pass has higher amplification efficiency, is more cost effective and is more flexible. In the small signal regime, considered in our work, experimental results in the existing literature have shown that the double-pass amplifier is clearly superior to the single-pass amplifier (see also [14], [15]). We use the rate equations derived in Desurvire's paper [18] to study the dynamic amplification for the double-pass configuration. The signal, ASE, and pump power propagating along the fiber can be written as

$$
\begin{aligned}
& \frac{d P_{s}^{ \pm}\left(z, \nu_{i}\right)}{d z}= \pm\left\{G_{s}\left(z, \nu_{i}\right)\left[P_{s}^{ \pm}\left(z, \nu_{i}\right)+P_{0}\right]\right.\left.-\alpha_{s}\left(z, \nu_{i}\right) P_{s}^{ \pm}\left(z, \nu_{i}\right)\right\} \\
& \frac{d P_{p}(z)}{d z}=-\alpha_{p}(z) P_{p}(z) .
\end{aligned}
$$

In (18) and (19), $P_{s}^{ \pm}\left(z, \nu_{i}\right), i=1,2, \ldots, N$, represents signal and ASE power, which is sliced into $N$ slots with arbitrary width $\Delta \nu$ centered at frequency $\nu_{i}$. The sign $(+)$ corresponds to signal or ASE power propagating in the same direction as the pump while the sign (-) indicates the direction opposite to the pump. $P_{p}(z)$ represents the pump power. The symbol $P_{0}$ represents an equivalent input noise power (cf. [19]). The symbols $\alpha, G$ represent absorption and gain coefficients, respectively.

The absorption and gain parameters of the Erbium doped single mode PM (polarization-maintaining) fiber used in this experiment were provided by the fiber manufacture. For simplicity, a trapezoidal shape of the Fiber Bragg Grating (FBG) 


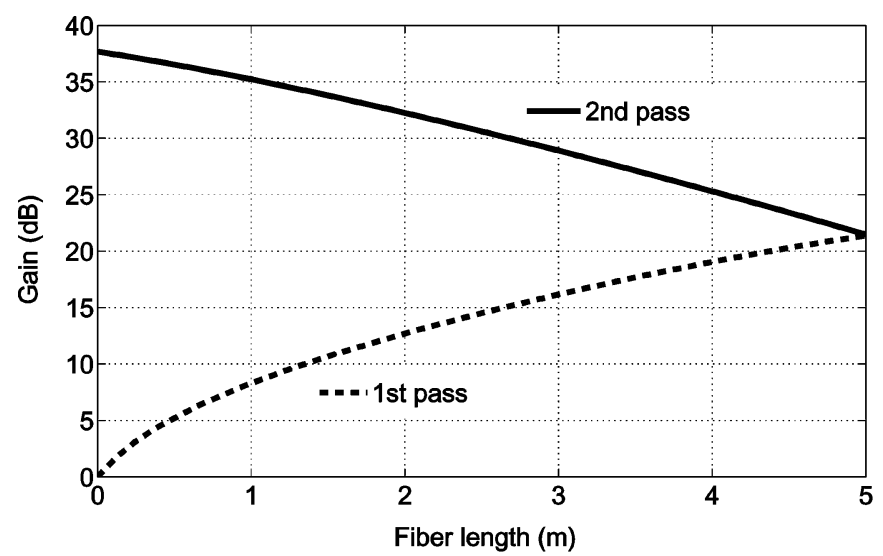

Fig. 4. Simulation results of the double-pass preamplifier with $-30 \mathrm{dBm}$ input power. The dashed line is the small signal gain for the signal propagating along the fiber. The solid line is the gain of the second pass.

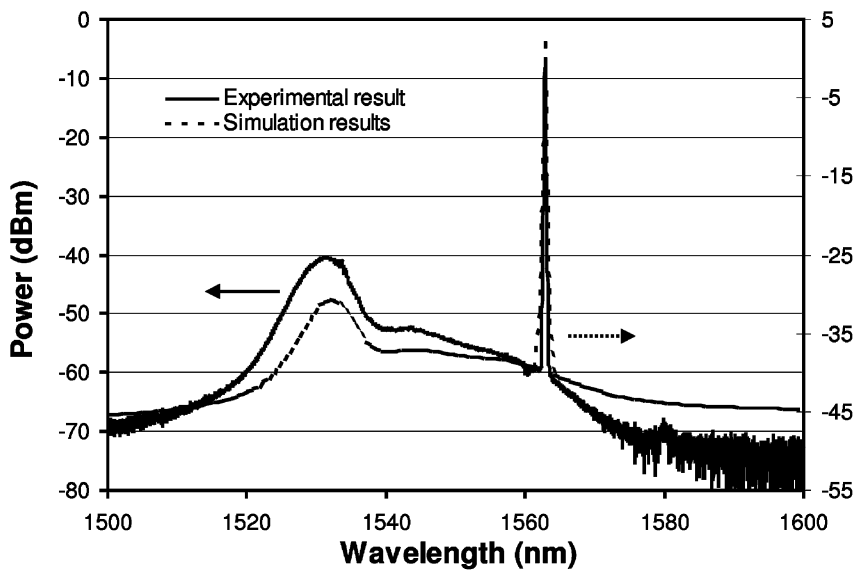

Fig. 5. Experimental and simulation results of double-pass optical amplifier. The solid line shows the experimental results and the dashed line shows the simulation results.

with $1 \mathrm{~nm}$ bandwidth at $3 \mathrm{~dB}$ and $20 \mathrm{~dB}$ contrast ratio is used for the simulation. Fig. 4 shows the small signal gain with $-30 \mathrm{dBm}$ input power. The dashed line represents the forward small signal gain propagating along the gain medium fiber. The solid line represents the backward signal gain. Simulation results also show that the double-pass optical amplifier design can efficiently suppress ASE (see Fig. 5).

\section{EXPERIMENTS}

\section{A. Experimental Setup and Characterization}

The optically preamplified coherent detection with time-domain filtering is investigated experimentally. The experimental setup is shown in Fig. 6. A $20 \mathrm{~mW}$ distributed feedback (DFB) polarization-maintaining (PM) laser diode (JDS Uniphase) is used as the seed source. The linewidth is less than $1 \mathrm{MHz}$. The wavelength of the seed source is shifted by $500 \mathrm{MHz}$ in the first-order output port of a polarization-maintained acousto-optic (AO) shifter (Brimrose). Two zero-chirp intensity Mach-Zehnders modulators (MZM) made by COVEGA are used in the experiments. One is the Mach-10 TM 004 integrated with a variable optical attenuator (VOA). This Mach-Zehnder is used to modulate the shifted laser beam and generate signal pulses. The other Mach-Zehnder, a Mach-10 056, is used for time-domain filtering. The unshifted $\mathrm{CW}$ laser from the zero-order output port of the $\mathrm{AO}$ shifter is used as a local oscillator connecting to the PM $3 \mathrm{~dB}$ mixer.

An analog modulator bias is used to supply the bias voltages on both modulators. The integrated VOA of the Mach-Zehnder of the Mach-10 TM 004 is adjusted by a DC power supplier. Both Mach-Zehnders have a $10 \mathrm{GHz}$ bandwidth. DG535 digital delay/pulse generator made by Stanford Research Systems, Inc. is used to drive the first Mach-Zehnder to generate the signal pulses. The pulse generator is also used to synchronize the second pulse generator (Philips, PM5786B), which supplies the pulses on the second Mach-Zehnder. In order to simulate measuring the return signal, two external in-line PM VOAs are used to control the signal strength up to $60 \mathrm{~dB}$ variations. A $3 \mathrm{~dB}$ PM coupler is used to mix signal and local oscillation light. A PIN photodiode (Discovery Semiconductors, Inc.) is chosen to detect the mixed light. The photodiode has a $10 \mathrm{GHz}$ bandwidth and the optical power linearity range can be up to $25 \mathrm{~mW}$, which ensures that the $25 \mathrm{~mW}$ local oscillator optical power is still within the photodiode linear responsive range.

An $R C$ circuit is used to filter out the DC component that is mainly contributed by the local oscillator. Two Mini Circuit ZFL-1000LN amplifiers are used to amplify the electrical signal passing through the $R C$ filter. The amplifier has a $1 \mathrm{GHz}$ bandwidth and a $2.9 \mathrm{~dB}$ noise figure. A $2.5 \mathrm{GHz}$ Tektronix digital phosphor oscilloscope (TDS7254B) is used to do signal capturing and processing. A LabVIEW program was developed to control the oscilloscope. The program is integrated with special signal processing functions such as bandpass filtering, squaring, averaging, summing, and so on.

\section{B. Optical Preamplifier}

In accordance with our earlier simulation results on the $\mathrm{Er}$ doped fiber [10], fibers of length 4, 5, 6, and $7.5 \mathrm{~m}$ were used in the experiment. For the single-pass amplifier, $7.5 \mathrm{~m}$ demonstrated the best performance, whereas the double-pass amplifier had the best performance with a $5 \mathrm{~m}$ gain medium fiber, which is one third shorter than the single-pass optimum length. A $100 \mathrm{GHz}$ bandwidth and $20 \mathrm{~dB}$ contrast ratio PM FBG was specially designed for this experiment. By using this FBG and combining with a PM circulator, a reflector with $20 \mathrm{~dB}$ difference between in-band and out-of-band spectrum was successfully constructed. The return signal gain $20 \mathrm{~dB}$ refers to those out-of-band spectrum components only at the end of the gain medium fiber.

To achieve the maximum signal power, the input signal wavelength was tuned to the central wavelength of the FBG by changing the temperature of the seed laser diode. Fig. 7 shows the forward single-pass and backward double-pass spectra. The filtered signal and in-band ASE was $20 \mathrm{~dB}$ higher than the peak of any other wavelength of the ASE. The experimental and simulation results of the double-pass preamplifier matched well, as can be seen in Fig. 5. To further reduce the backward ASE, another pair of FBG and circulator was used in the system as the bandpass filter (see Fig. 6). Although most of the ASE can be successfully removed by the optical bandpass filter and the narrowband reflector, the residual in-band ASE still remains. To reduce this residual ASE, the second MZM is used 


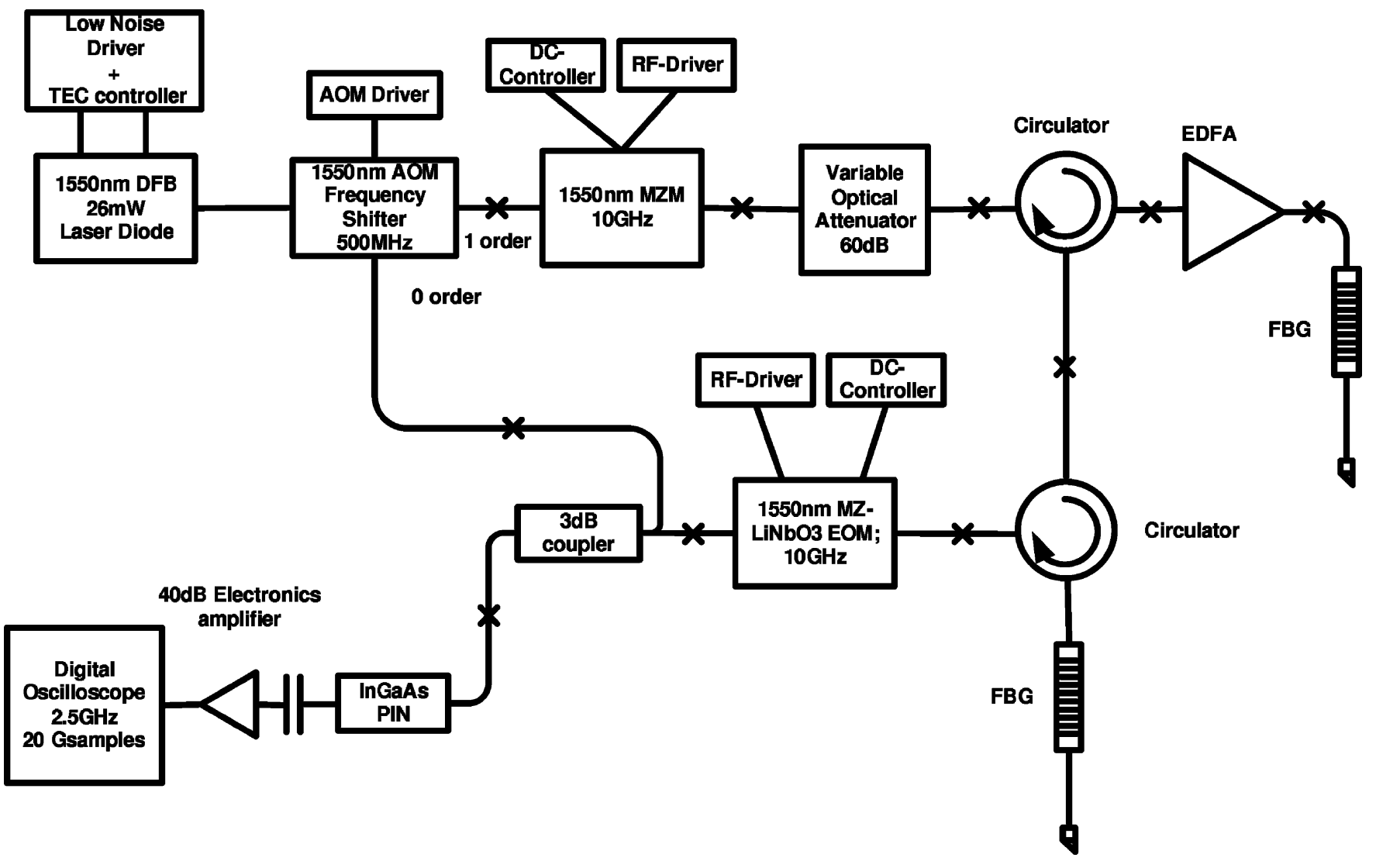

Fig. 6. Schematic diagram of a double-pass preamplified coherent detection system with time-domain filtering.

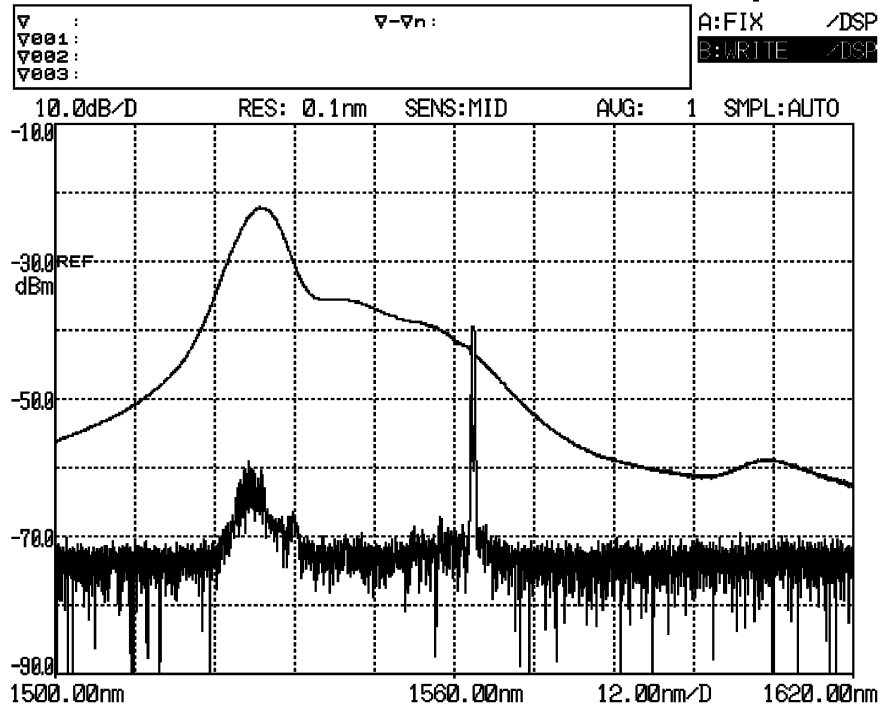

Fig. 7. Output spectrum of the optical preamplifier with $100 \mathrm{pW}$ input signal. The top curve is the forward ASE and the bottom curve is the filtered backward signal and in-band ASE.

as a time-domain filter that is modulated by a Philips pulse generator synchronized with the first MZM.

Fig. 8 shows the experimental results with time-domain filters and three duty cycles: $5 \%, 20 \%$, and $100 \%$. For the $5 \%$ and $20 \%$ duty cycle cases, the open time slots are $100 \mathrm{~ns}$ and $400 \mathrm{~ns}$, respectively, for a $500 \mathrm{kHz}$ repetition rate. For the $100 \%$ duty cycle case, the TDF is totally open. The average received

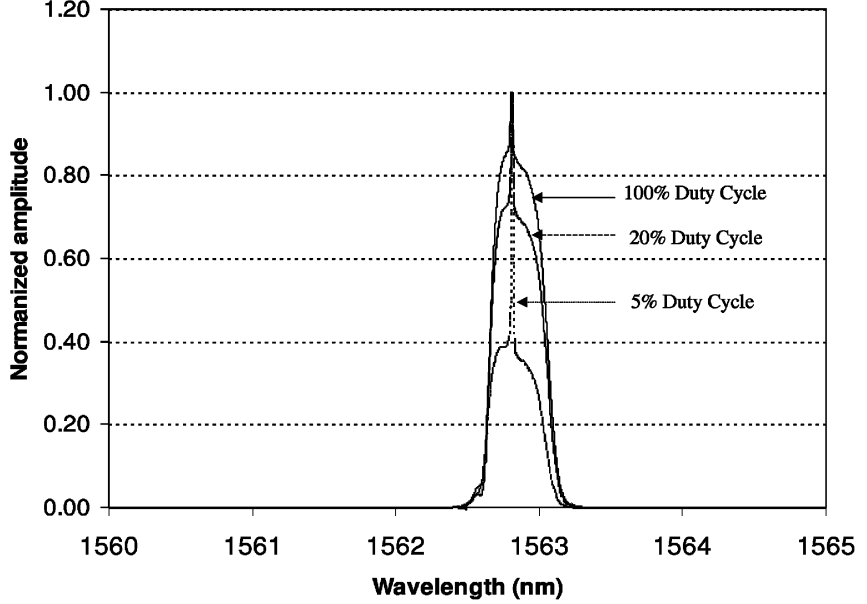

Fig. 8. The spectra of the signal and in-band ASE. The solid curve shows the spectrum obtained with $100 \%$ duty cycle, which means the time window of the TDF is totally open. The short dashed curve shows the spectrum obtained by using a $20 \%$ duty cycle. The long dashed curve shows the spectrum obtained with a $5 \%$ duty cycle.

power is $100 \mathrm{pW}$. The experimental results show that the difference from the signal peak to the pedestal was improved from $0.7 \mathrm{~dB}$ for $\mathrm{CW}$ to $1.5 \mathrm{~dB}$ and $4.25 \mathrm{~dB}$ for $20 \%$ and $5 \%$ duty cycles, respectively. Since the spectra in Fig. 8 were captured by an Optical Spectrum Analyzer (OSA), we have shown that the time-domain filter is a very efficient method to improve direct detection sensitivity. We note that the insertion loss introduced by the TDF is approximately $3.45 \mathrm{~dB}$. However, the TDF does 
not appreciably affect the signal-to-noise ratio, since the same loss is applied to both the signal and the noise.

\section{Coherent Detection Experiment}

With the experimental setup in Fig. 6, the preliminary experiment successfully demonstrated fiber-based heterodyne detection. The signal pulses are generated by the first MZM with pulse duration of $10 \mathrm{~ns}$ and a $500 \mathrm{kHz}$ repetition rate. With the same detection system, we also evaluate the direct detection performance and find that the sensitivity of direct detection is over $20 \mathrm{~dB}$ less than that of coherent detection, even though the direct optical detection of on-off keyed signals is better, in principle, than that of heterodyne detection of these same signals. In practice, however, thermal noise, excess noise, and electronics background radiation seriously degrade the direct detection sensitivity. As discussed in [20], a practical implementation of a direct detection system has performance at least 10 to $30 \mathrm{~dB}$ below the theoretical limit.

From (17), we can see that the received signal can be amplified by the local oscillation after two-beam mixing. If the local oscillation is strong enough, the shot noise caused by the local oscillation will dominate the noise field. According to this experimental condition, the local oscillator should be higher than $1 \mathrm{~mW}$ to overcome the Johnson noise. In our previous work [10], by using a single-pass optical preamplifer, the lowest detectable power threshold of coherent detection achieved was $0.7 \mathrm{pW}$, corresponding to 11 photons within the $10 \mathrm{~ns}$ pulse width. For the double-pass preamplifier, the lowest detectable power was $0.5 \mathrm{pW}$, corresponding to 8 photons. We remark that in lidar applications, detector performance is evaluated in terms of the number of detectable photons, whereas in communication applications performance is typically evaluated in terms of bit error rate (cf. [1]).

The experimental results of the detection performance with the different time-domain filtering windows are shown in Fig. 9. The results, shown with solid square, diamond and circle markers, were obtained using a digital Hamming bandpass filter of bandwidth $200 \mathrm{MHz}$, centered at $500 \mathrm{MHz}$. As expected, the detection performance improves when the time-domain filter window is made more narrow. Compared with an open window, $100 \mathrm{~ns}$ or $5 \%$ duty cycle and $50 \%$ duty cycle time windows show $2 \mathrm{~dB}$ and $1 \mathrm{~dB}$ improvements, respectively, for a single shot process at $10 \mathrm{pW}$ input power level. The shot noise limits in the theoretical analysis for the bandwidths of $200 \mathrm{MHz}$ and $1 \mathrm{GHz}$ are also given Fig. 9 as solid and short dashed curves, respectively. Both experimental and analytical results show that the redundant digital bandpass filter is very helpful in improving the detection performance.

In Fig. 9, the best experimental results that are close to the shot noise were obtained using the $5 \%$ duty cycle time window at a bandwidth of $200 \mathrm{MHz}$. However, there is not a significant change when the time window is changed from $20 \%$ to $50 \%$ duty cycle at $1 \mathrm{GHz}$ digital bandwidth. As mentioned before, a wider bandwidth of the digital bandpass filter results in stronger shot noise. In this case, the shot noise is the dominant noise source for the system. Besides, the ASE induced noise is limited, since only a small portion of the ASE passes through the narrow and strong optical bandpass filter. Only half of this ASE has the same polarization as the signal and local oscillation. Even in

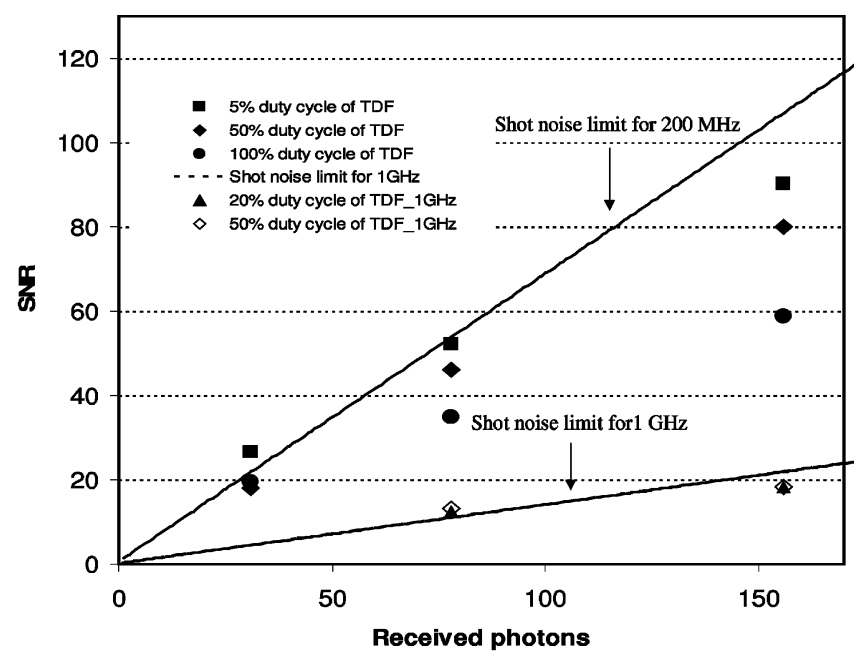

Fig. 9. Signal-to-noise ratio versus number of received photons with different detection conditions. The solid line represents the shot noise with a digital bandpass filter of $200 \mathrm{MHz}$ and the dashed one corresponds to $1 \mathrm{GHz}$ bandwidth.

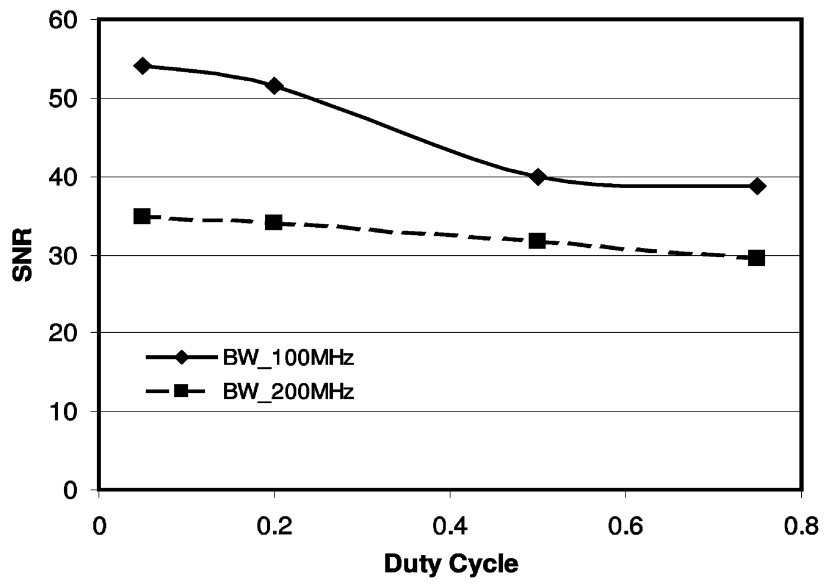

Fig. 10. SNR versus duty cycle (or time window size) of the time-domain filter by averaging 50 independent traces. The solid curve is measured with a 100 $\mathrm{MHz}$ bandwidth bandpass filter, while the dashed curve is measured with a bandwidth of $200 \mathrm{MHz}$.

this half of the ASE, there is a small portion that has a coherent relationship with the signal and local oscillation.

Since all the measurements are very sensitive to the environment, multiple traces are averaged to evaluate the detection sensitivities. In addition, the slight changes in the polarization of the light can also introduce additional variations. Averaging multiple traces smoothes out the effects of the random noise to some extent. Meanwhile, the inherent jitter from the measurement equipment can also reduce the peak of the signal. Fig. 10 shows the SNR as a function of the duty cycle of the time-domain filter with $100 \mathrm{MHz}$ and $200 \mathrm{MHz}$ bandwidths with $10 \mathrm{pW}$ average received power, corresponding to 156 photons. For the fixed repetition rate, the higher duty cycle results in a wider time window of the time-domain filter. Fig. 10 clearly shows that the time-domain filter is helpful in improving the coherent detection sensitivity, even though the improvement is not as noticeable as in the case of direct detection. The figure also shows that narrowing the bandwidth can improve the sensitivity significantly. However, when the bandwidth of the digital filter is wide enough such that shot noise becomes the dominant noise source, the detection sensitivity does not improve appreciably. 


\section{CONCLUSION}

We demonstrated a novel fiber-based coherent detection system that uses a double-pass optical preamplifier, a bandpass filter and a time-domain filter to strengthen the received signal and reduce ASE effects. We described the basic concept and principle and derived related equations for this detection architecture. We presented simulation results for the low noise figure double-pass optical preamplifer. With a $100 \mathrm{GHz}$ optical bandpass filter, we conducted experiments with different window sizes for the time-domain filter. We successfully demonstrated $2 \mathrm{~dB}$ and $1 \mathrm{~dB}$ SNR improvements with respect to open windows, i.e., $100 \%$ duty cycle with $100 \mathrm{~ns}$ and $1000 \mathrm{~ns}$ window size or 5\% duty cycle and 50\% duty cycle, respectively, using a digital bandpass filter with $200 \mathrm{MHz}$ bandwidth. We also demonstrated that for a shot noise-dominated optically preamplified coherent system, the time-domain filter could not improve the detection performance significantly. When the ASE-induced noise is comparable to the shot noise, the time-domain filter substantially improves the performance of coherent detection.

The results presented in this paper show that the proposed system can significantly improve the performance of lidar applications. Besides lidar, the double-pass preamplified TDF coherent system could also be applied to digital communication systems. In this case, bit error rate (BER) measurements should be taken to evaluate the system performance.

\section{REFERENCES}

[1] M. Liu, Principles and Applications of Optical Communications. New York: McGraw-Hill, 1996.

[2] U. Timor and R. A. Linke, "A comparison of sensitivity degradations for optical homodyne versus direct detection of on-off keyed signals," IEEE J. Lightw. Technol., vol. 6, no. 11, pp. 1782-1788, Nov. 1988.

[3] P. M. Gabla, E. Leclerc, and C. Coeurjolly, "Practical implementation of a highly sensitive receiver using an erbium-doped fiber preamplifier," IEEE Photon. Technol. Lett., vol. 3, no. 8, pp. 727-729, Aug. 1991.

[4] P. P. Smyth, R. Wyatt, A. Fidler, P. Eardley, A. Sayles, and S. CraigRyan, " 152 photons per bit detection at $622 \mathrm{Mbit} / \mathrm{s}$ to $2.5 \mathrm{Gbit} / \mathrm{s}$ using an erbium fiber preamplifier," J. Electric. Lett., vol. 26, no. 19, pp. 1604-1605, Sep. 1990.

[5] O. K. Tonguz and R. E. Wagner, "Equivalence between preamplified direct detection heterodyne receivers," IEEE Photon. Technol. Lett., vol. 3, no. 9, pp. 835-837, Sep. 1991.

[6] H. Sanjoh, K. Tsuzuki, and Y. Yoshikuni, "Time-domain filtering to reduce signal-spontaneous beat noise for a pulse type multiwavelength light source," IEEE Photon. Technol. Lett., vol. 15, no. 5, pp. 757-759, May 2003.

[7] B. Glance, G. Eisenstein, P. J. Fitzgerald, K. J. Pollock, and G. Raybon, "Sensitivity of an optical heterodyne receiver in presence of an optical preamplifier," Electron. Lett., vol. 24, no. 19, pp. 1229-1230, Sep. 1988.

[8] S. Ryu and Y. Horiuchi, "Use of an optical amplifier in a coherent receiver," IEEE Photon. Technol. Lett., vol. 13, no. 7, pp. 663-665, Jul. 1991.

[9] P. Theodorou and J. M. H. Elmirghani, "Selective multi-channel coherent optically preamplified systems: Sensitivity assessment," in IEEE Globecom, Nov. 1997, vol. 1, pp. 275-279.

[10] Y. Chen, W. Torruellas, B. L. Mark, R. Burnham, and H. Verdun, "Analysis of fiber-based coherent detection employing optical preamplifier and time-domain filter," in Photonic Sensing Technologies, Proc. SPIE, Oct. 2006, vol. 6371, pp. 63710D.1-63710D.10.

[11] S. Hwang, K. W. Song, H. J. Kwon, J. Koh, Y. J. Oh, and K. Cho, "Broad-band erbium-doped fiber amplifier with double-pass configuration," IEEE Photon. Technol. Lett., vol. 13, no. 12, pp. 1289-1291, Dec. 2001.
[12] S. W. Harun, N. Tamchek, P. Poopalan, and H. Ahmad, "Double-pass L-band EDFA with enhanced noise figure characteristics," IEEE Photon. Technol. Lett., vol. 15, no. 8, pp. 1055-1057, Aug. 2003.

[13] M. Z. Jamaludin, F. Abdullah, F. A. Rahman, M. A. Mahdi, and M. K. Abdullah, "Performance enhancement of transmission system using double-pass EDFA with built-in variable optical Bragg grating," in Proc. IEEE Int. Conf. Telecommun. Malaysia Int. Conf. Commun., May 2007, pp. 43-46.

[14] A. W. Naji, M. S. Z. Abidin, M. H. Al-Mansoori, M. Z. Jamaludin, M K. Abdullah, S. J. Iqbal, and M. A. Mahdi, "Dual-function remotelypumped erbium-doped fiber amplifier: Loss and dispersion compensator," Opt. Express, vol. 14, no. 8, pp. 8054-8059, Sep. 2006.

[15] B. Bouzid, M. K. Abdulla, and M. A. Mahdi, "High-gain erbium-doped fiber amplifier incorporating a double-pass amplification technique as a preamplifier," Laser Phys., vol. 18, no. 4, pp. 460-463, Apr. 2008.

[16] L. Mandel and E. Wolf, Optical Coherent and Quantum Optics. Cambridge, U.K.: Cambridge Univ. Press, 1995.

[17] P. J. Winzer and A. Kalmar, "Sensitivity enhancement of optical receivers by impulsive coding," IEEE J. Lightw. Technol., vol. 17, no. 2, pp. 171-177, Feb. 1999

[18] E. Desurvire and J. R. Simpson, "Amplification of spontaneous emission in erbium-doped single-mode fibers," IEEE J. Lightw. Technol., vol. 7, no. 5, pp. 835-845, May 1989.

[19] E. Desurvire, Erbium Doped Fiber Amplifiers-Principles and Applications. New York: Wiley, 1994.

[20] E. Rochat, R. Dändliker, K. Haroud, R. H. Czichy, U. Roth, D. Costantini, and R. Holzner, "Fiber amplifiers for coherent space communication," IEEE J. Sel. Topics Quantum Electron., vol. 7, pp. 64-80, Jan./Feb. 2001.

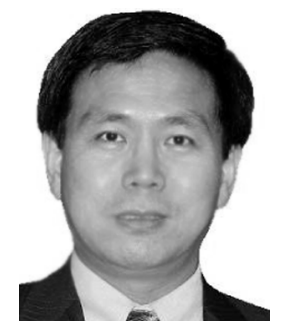

Youming Chen received the B.A.Sc. degree in laser physics from the Department of Optics, Shandong University, China, in 1984, the M.Sc. degree in laser physics from the Shanghai Institute of Optics and Fine Mechanics, Chinese Academy of Sciences, China, in 1988. He received the Ph.D. degree in information technology and engineering from George Mason University, Fairfax, VA, in 2008.

He was an Assistant Professor at the Shanghai Institute of Optics and Fine Mechanics from 1989 to 1994. He was a visiting scholar at Howard University, USA, from 1994 to 1996. He joined Science and Engineering Service, Inc. as a Laser Scientist in 1996. He was a Laser Scientist at Fibertek, Inc. from 1997 to 2000. In 2000, he joined Corvis (now Level 3 Communications) as a Senior Optical Engineer II. Since 2002, as a Senior Laser Scientist, he has been working with Fibertek, Inc. His current research interests are mainly in design and development of novel high power coherent fiber lasers and advanced fiber based lidar applications. He has authored or coauthored 60 journals and conferences papers in laser physics and opto-electronics.

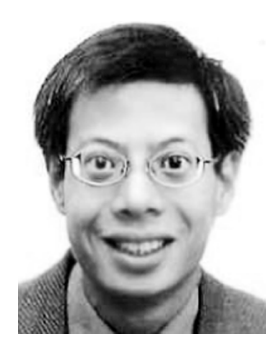

Brian L. Mark (M'91-SM'08) received the B.A.Sc. degree in computer engineering with an option in mathematics from the University of Waterloo, Canada, in 1991 and the Ph.D. degree in electrical engineering from Princeton University, Princeton, NJ, in 1995.

He was a Research Staff Member at the C\&C Research Laboratories, NEC USA, from 1995 to 1999. In 1999, he was on part-time leave from NEC as a visiting researcher at Ecole Nationale Supérieure des Télécommunications in Paris, France. In 2000, he joined the Department of Electrical and Computer Engineering, George Mason University, Fairfax, VA, where he is currently an Associate Professor His research interests lie broadly in the design, modeling, and analysis of communication systems, computer systems, and communication networks.

Dr. Mark was a co-recipient of the Best Conference Paper Award at IEEE INFOCOM'97. He received a National Science Foundation CAREER Award in 2002. Currently, he serves as an Associate Editor for IEEE TRANSACTIONS ON VEHICULAR TECHNOLOGY. 


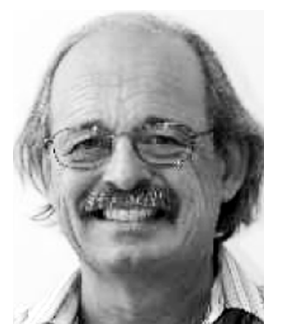

Ralph Burnham received the B.A.Sc. degree in physics from Georgia Tech, Atlanta, in 1966, and the Ph.D. degree from the University of Florida in 1972.

From 1972 to 1973 he served as a Post Doctoral Fellow at the National Bureau of Standards, Gaithersburg, MD, where he was with the Naval Research Laboratory, Laser Physics Branch, where he pioneered the development of several lasers including discharge-pumped are gas halide and mercury halide excimers, metal vapor Raman lasers, and the optically pumped $546 \mathrm{~nm}$ mercury vapor laser. Since joining Fibertek, Inc. in 1987, he has concentrated on the development of advanced solid-state lasers and laser detectors. His work in this area includes the design and analysis of laser systems and associated subsystems. His interest in laser technology centers on development of diode-pumped solid-state lasers for military and commercial applications. His research division at Fibertek has been at the cutting edge of solid-state laser $R \& D$ for over 20 years, and has fielded numerous diode-pumped lasers and transitioned advanced military lasers to production. He is currently a Senior Vice President of Fibertek, Inc., where he manages laser research and development.
Horacio Verdun (M'88) received the degree of Ingeniero Químico from the Facultad de Ingenierí Química, Universidad National del Litoral, Sante Fé, Argentina, in 1966. In 1971 he graduated from the Escuela de Graduados, Universidad Nacional de Ingeniería, Lima, Perú, with the degree of Magister en Física, and received the Ph.D. degree in physics from the University of Maryland in 1973 .

After leaving academia in 1979, where he had been a lecturer and researcher in physics for many years, he joined Science Applications, Inc., where he performed concept analysis for thermophotovoltaic solar energy conversion, designed and built pulsed high-flux thermal simulators, and analyzed, in collaboration with Dr. Walter Koechner, new concepts for high pulse energy and high average power diode-pumped solid state lasers. In 1985 he joined Fibertek, Inc., where he is a Senior Vice President. He has been in charge of research on new materials for solid state lasers and the development, design, fabrication, and fielding of several systems, such as very sensitive optical sensors, tunneling current probe for the contactless characterization of wafer-level focal plane arrays, mini-laser rangefinders and lidars for wire detection and biological agent detection. His current interests include the development of advanced fiber lasers and fiber optics based lidar systems. 